\title{
Doxorubicin-induced persistent oxidative stress to cardiac myocytes
}

\author{
Shaoyu Zhou a, Carlos M. Palmeira ${ }^{\mathrm{b}}$, Kendall B. Wallace ${ }^{\mathrm{a}, *}$ \\ a Department of Biochemistry and Molecular Biology, and Toxicology Graduate Program, School of Medicine, \\ University of Minnesota, Duluth, MN 55812, USA \\ ${ }^{\mathrm{b}}$ Center for Neurosciences and Cell Biology, Department of Zoology, University of Coimbra, Portugal
}

Received 11 December 2000; received in revised form 13 February 2001; accepted 15 February 2001

\begin{abstract}
We recently reported a cardioselective and cumulative oxidation of cardiac mitochondrial DNA (mtDNA) following subchronic administration of doxorubicin to rats. The mtDNA adducts persist for up to 5 weeks after cessation of doxorubicin treatment. Since the evidence suggests that this persistence of mtDNA adducts cannot be attributed to a lack of repair and replication, we investigated whether it might reflect a long-lasting stimulation of free radical-mediated adduct formation. Male Sprague-Dawley rats received weekly s.c. injections of either doxorubicin $(2 \mathrm{mg} / \mathrm{kg})$ or an equivalent volume of saline. Cardiac myocytes isolated from rats following 6 weekly injections of doxorubicin expressed a much higher rate of reactive oxygen species (ROS) formation compared to saline controls. This higher rate of ROS formation persisted for 5 weeks following the last injection. Associated with this was a persistent depression of GSH in heart tissue, while protein-thiol content was not markedly altered. These data suggest that the accumulation and persistence of oxidized mtDNA may be due, not to the stability of the adducts, but to some as yet undefined toxic lesion that causes long-lasting stimulation of ROS generation by doxorubicin. This persistent generation of ROS may contribute to the cumulative and irreversible cardiotoxicity observed clinically with the drug. (C) 2001 Elsevier Science Ireland Ltd. All rights reserved.
\end{abstract}

Keywords: Cardiac myocytes; Doxorubicin; Glutathione; Protein-thiol; Reactive oxygen species

\section{Introduction}

Long-term administration of doxorubicin (DOX), a potent antineoplastic agent for treating various human tumors, causes a cumulative dose-

\footnotetext{
* Corresponding author. Tel.: + 1-218-7268899; fax: + 1218-7268014.

E-mail address: kwallace@d.umn.edu (K.B. Wallace).
}

dependent cardiomyopathy. The mechanism of this DOX-induced cardiotoxicity is attributed to free radical generation, stimulation of lipid peroxidation, and subsequent alteration of cellular membrane integrity. This hypothesis is supported by the reported cytoprotective effect of antioxidants against DOX toxicity (Kimura et al., 2000; Wang and Kang, 1999; Wu and Kang, 1998). An increasing number of studies demonstrate that 
DOX causes a dose-dependent disruption of cardiac mitochondrial structure and bioenergetic function (Nicolay and De Kruijff, 1987; Papadopoulou et al., 1999; Praet and Ruysschaert, 1993; Solem et al., 1994, 1996), and implicate mitochondrial dysfunction in the pathogenesis of DOX-induced cardiomyopathy. We hypothesize that DOX-induced cardiotoxicity is mediated through altered mitochondrial function caused by DOXinduced free radical generation.

We recently demonstrated that DOX causes the preferential accumulation of 8-hydroxydeoxyguanosine (8OHdG) in cardiac mitochondrial DNA (mtDNA) (Palmeira et al., 1997). Moreover, this cardioselective oxidation of mitochondrial DNA is cumulative with repeated dosing and persists for 5 weeks after cessation of DOX treatment (Serrano et al., 1999). The accumulation and persistence of $8 \mathrm{OHdG}$ caused by DOX is in accord with the irreversible cardiomyopathy observed clinically. Since expression of the mitochondrial genome is essential to the integrity of the respiratory chain, oxidation of mtDNA may interfere with the expression of critical mitochondrial electron transport proteins, which might underlie the pathogenesis of DOX cardiomyopathy.

The formation of $8 \mathrm{OHdG}$ adducts is attributed to reactive oxygen species. Indeed, a strong correlation has been characterized between the rate of generation of hydroxyl free radicals in submitochondrial particles and the occurrence of $8 \mathrm{OHdG}$ adducts to the mitochondrial genome (Giulivi et al., 1995). Although a number of initial studies reported deficiencies in mtDNA repair mechanisms that might explain the persistence of mtDNA adducts (LeDoux et al., 1992; Miyaki et al., 1977; Niranjan et al., 1981; Stairs et al., 1983), recent evidence demonstrates that mitochondria are capable of repairing oxidative damage to mtDNA (Salazar and Van Houten 1997; Yakes and Van Houten, 1997). Moreover, mtDNA adducts can be eliminated by mitochondrial turnover. Thus, the persistence of mtDNA adducts is not likely attributable to the stability of the adducts in vivo. Therefore, we questioned whether the persistence of $8 \mathrm{OHdG}$ adducts might reflect prolonged stimulation of ROS generation and oxidative stress that continues long after termination of drug treatment.

\section{Materials and methods}

\subsection{Materials}

Doxorubicin was purchased from Pharmacia \& Upjohn Co. (Kalamazoo, MI). 5-(and 6-)chloromethyl-2',7'-dichlorodihydro-fluorescein diacetate (CM- $\mathrm{H}_{2}$ DCFDA) was purchased from Molecular Probes (Eugene, OR). All other chemicals were of analytical grade and obtained from common commercial sources.

\subsection{Animals}

Male Sprague-Dawley rats (Harlan Labs, Madison, WI) weighing $175 \pm 5 \mathrm{~g}$ were divided randomly between treatment groups and maintained in AAALAC-accredited, climate-controlled facilities and allowed free access to food (Purina Chow) and water. Rats received 6 weekly s.c. injections of either doxorubicin $(2 \mathrm{mg} / \mathrm{kg})$ or an equivalent volume of saline $(1 \mathrm{ml} / \mathrm{kg})$. The animals were killed by decapitation 1 or 5 weeks after the last injection. The hearts were excised immediately to isolate cardiac myocytes for determination of ROS generation or frozen in liquid nitrogen and stored at $-80^{\circ} \mathrm{C}$ until assayed for GSH and protein-thiol.

\subsection{Determination of reactive oxygen species (ROS)}

ROS were determined fluorometrically using a Labsystems type 374 plate-reader fluorometer. Cardiac myocytes were isolated as previously described (Solem et al., 1996). Isolated cardiac myocytes were suspended in Rosewell Park Memorial Institute (RPMI) medium at a density of $5 \times 10^{5}$ live cells $/ \mathrm{ml}$. Cells were loaded with 50 $\mu \mathrm{M} \mathrm{CM}-\mathrm{H}_{2}$ DCFDA (prepared in DMSO) for 15 min at $37^{\circ} \mathrm{C}$. Cell suspension $\left(200 \mu \mathrm{l} ; 10^{5}\right.$ cells $)$ were loaded into a 96-well plate. The formation of the oxidized derivative of $\mathrm{CM}-\mathrm{H}_{2} \mathrm{DCF}$, was monitored fluorimetrically at an excitation wavelength 
$485 \mathrm{~nm}$ and an emission wavelength $538 \mathrm{~nm}$. The fluorescence was monitored for $30 \mathrm{~min}$ to calculate the rate of ROS formation. The results were expressed as fluorescence intensity increase per $10^{5}$ cells per $10 \mathrm{~min}$.

\subsection{Analysis of GSH and protein-thiol}

Determination of GSH was performed by a modified fluorometric method (Hissin and Hilf, 1976). Rat heart tissue (250 mg) was homogenized on ice using a Polytron homogenizer. The solution used for homogenization consisted of $3.75 \mathrm{ml}$ of phosphate-EDTA buffer $(0.1 \mathrm{M}$ sodium phosphate, $5 \mathrm{mM}$ EDTA, $\mathrm{pH} 8.0$ ) and $1 \mathrm{ml}$ of $25 \%$ $\mathrm{HPO}_{3}$. The total homogenate was centrifuged at $4^{\circ} \mathrm{C}$ at $100000 \times g$ for $30 \mathrm{~min}$. The supernatant was removed for the assay of GSH, and the pellet was used for determining protein-thiol content. For the GSH assay, $10 \mu \mathrm{l}$ of the $100000 \times g$ supernatant was added to $1.89 \mathrm{ml}$ of phosphate-EDTA buffer, and $100 \mu \mathrm{l}$ of $1 \mathrm{mg} / \mathrm{ml} o$-phthalaldehyde (OPT) solution (prepared in methanol). After thorough mixing and incubation at room temperature for 15 $\min , 250 \mu 1$ of the solution was loaded into a 96-well plate. Fluorescence at $405 \mathrm{~nm}$ was measured with the excitation at $320 \mathrm{~nm}$. Quantification of GSH was obtained by employing commercial GSH standards. The results are expressed as $\mu$ mole GSH per gram wet heart weight.

Determination of protein-thiol was performed by a modification of the method of Riddles et al. (1979). Briefly, the $100000 \times g$ pellet was resuspended in $2 \mathrm{ml}$ phosphate buffer. To $0.5 \mathrm{ml}$ of this mixture, $4.5 \mathrm{ml}$ phosphate buffer and $50 \mu \mathrm{l}$ of 10 $\mathrm{mM}$ 5,5'-dithiobis-(2-nitrobenzoic acid) (DTNB) was added. After thorough mixing and incubation at room temperature in the dark for $15 \mathrm{~min}$, the solution was transferred to a cuvette. Absorbance was measured at $412 \mathrm{~nm}$ using a Beckman DU 7400 spectrophotometer. The results are expressed as $\mu$ mole 2-nitro-5-thiobenzoic acid (TNB) per gram wet heart weight using a coefficient of $13600 \mathrm{M}^{-1}$ $\mathrm{cm}^{-1}$.

\subsection{Statistical analysis}

All data are expressed as the mean $\pm \mathrm{SE}$ for two-five separate animals. Differences between DOX and saline treatment were analyzed using the Student $t$ test. A probability of $P<0.05$ served as the criterion for statistical significance.

\section{Results}

The initial weights for saline and DOX groups were $176 \pm 4$ and $175 \pm 2 \mathrm{~g}$, respectively. As shown in Table 1, 6 weeks of DOX treatment retarded rat body weight gain by about $10 \%$ compared to controls. This was associated with a $15 \%$ decrease in ventricular heart weight as compared to salinetreated rats but there was no difference in the heart weight to body weight ratio between DOX-treated and saline-treated rats. Moreover, the decrease in both body weight and heart weight of DOX-treated rats persisted for a 5 week period after cessation of DOX treatment: DOX rats maintained body weight, while control rats continued to gain weight (Table 1).

The yield and viability of cardiac myocytes were not different between 6 week DOX and salinetreated rats. However, both cell yield and cell viability decreased markedly after 5 weeks recovery following the sixth injection (cell yields

Table 1

Rat body and heart weight $(\mathrm{g})^{\mathrm{a}}$

\begin{tabular}{lcc}
\hline & Saline & DOX \\
\hline $6+1^{\text {b }}$ & & \\
Body weight $(\mathrm{Bw})$ & $387 \pm 29$ & $346 \pm 23^{\mathrm{c}}$ \\
Heart weight $(\mathrm{Hw})$ & $1.66 \pm 0.12$ & $1.42 \pm 0.06^{\mathrm{c}}$ \\
Ratio $(\mathrm{Hw} / \mathrm{Bw}) \times 100$ & $0.43 \pm 0.01$ & $0.41 \pm 0.01$ \\
$6+5 \mathrm{w}^{\mathrm{b}}$ & & \\
Body weight $(\mathrm{Bw})$ & $420 \pm 2$ & $320 \pm 4^{\mathrm{c}}$ \\
Heart weight $(\mathrm{Hw})$ & $1.57 \pm 0.05$ & $1.22 \pm 0.09^{\mathrm{c}}$ \\
Ratio $(\mathrm{Hw} / \mathrm{Bw}) \times 100$ & $0.37 \pm 0.02$ & $0.38 \pm 0.04$ \\
\hline
\end{tabular}

\footnotetext{
${ }^{\text {a }}$ Data represent the mean \pm SE for four animals.

${ }^{\mathrm{b}} 6+1 \mathrm{w}$ and $6+5 \mathrm{w}$ represent samples from rats killed 1 or 5 weeks, respectively, following the last of 6 weekly injections of DOX or saline.

c Significant difference compared to saline control at $P<$ 0.05 .
} 


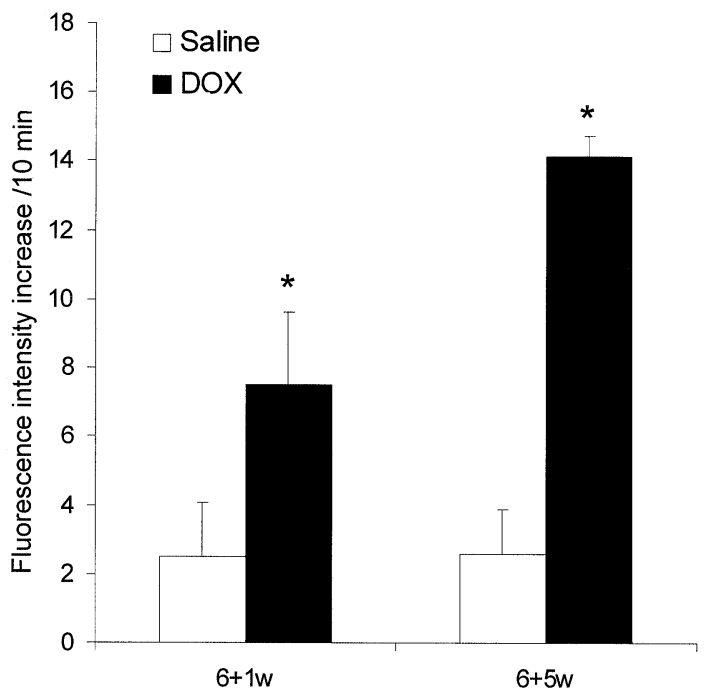

Fig. 1. DOX-induced ROS formation in cardiac myocytes. Rats received 6 weekly injections of DOX $(2 \mathrm{mg} / \mathrm{kg})$ or saline. $6+1 \mathrm{w}$ and $6+5 \mathrm{w}$ represent rats killed 1 or 5 weeks, respectively, after the last injection. Reactive oxygen species (ROS) were estimated fluorometrically using the probe CM$\mathrm{H}_{2}$ DCFDA, and expressed as fluorescent intensity increase per $10^{5}$ cells per $10 \mathrm{~min}$. Data represent the mean $\pm \mathrm{SE}$ of measurements from three $6+1 \mathrm{w}$ rats and two $6+5 \mathrm{w}$ rats, respectively. ${ }^{*}$, significant difference compared to saline control at $P<0.05$.

for saline and DOX-treated rats are $4.1 \pm 0.2 \times$ $10^{6}$ and $4.4 \pm 1.9 \times 10^{5}$ live cells per animal, with a viability of $74.5 \pm 1.5$ and $58.5 \pm 3.5 \%$, respectively). Myocytes isolated from rats treated for 6-weeks with DOX, expressed a dramatically higher rate of ROS formation compared to control (about three fold). Interestingly, the higher rate of ROS formation was evident even 5 weeks following the last injection of DOX (Fig. 1).

The effect of DOX on cardiac GSH is presented in Fig. 2. GSH in heart tissue from 6-week DOXtreated rats was significantly diminished, about $20 \%$ less compared to saline controls. The GSH content remained lower than control 5 weeks after discontinuation of DOX treatment (Fig. 2). There was no statistical difference in cardiac GSH content between the two control groups.

The effect of DOX on protein-thiol is presented in Fig. 3. No differences in protein-thiol content were found in heart tissues between DOX and saline-treated rats.

\section{Discussion}

In agreement with our previous findings (Solem et al., 1994, 1996), both body weight and heart weight were significantly less in 6-week DOXtreated rats compared to controls. Moreover, in the present study we found that the decrease in both body weight and heart weight persisted for a 5-week period after cessation of DOX treatment. This suggests an irreversible, and possibly progressive pathogenic process.

In correlation with the effect on body weight gain, we found a persistent stimulation of ROS formation. This persistent ROS formation is of particular importance in elucidating the mechanism of DOX-induced cardiotoxicity, as it correlates well with our recent finding of the persistence of oxidized mtDNA adducts. The accumulation of mtDNA 8OhdG adducts has been ascribed to ROS generation (Giulivi et al., 1995). Despite initial reports of deficiencies in mtDNA repair enzymes, recent studies demonstrate that oxidative DNA damage can be repaired in mito-

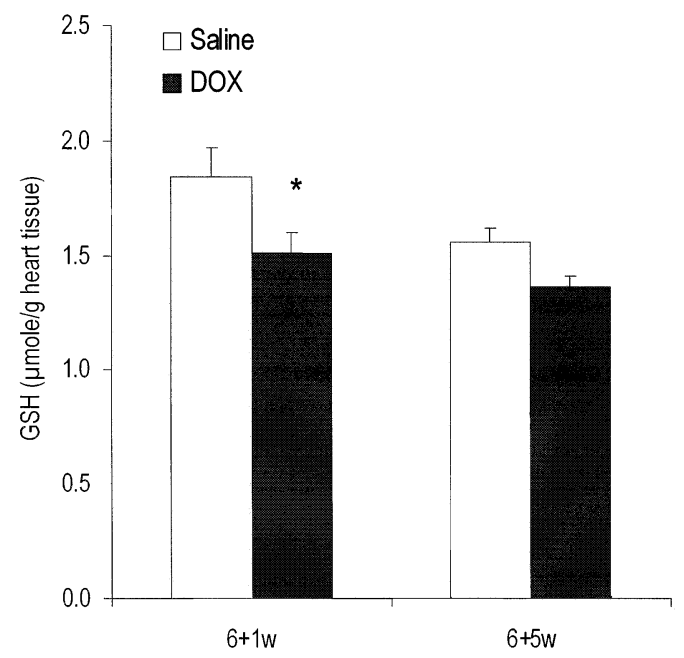

Fig. 2. GSH content in heart tissue harvested from DOX/saline treated rats. Rats received 6 weekly injections of DOX (2 $\mathrm{mg} / \mathrm{kg}$ ) or saline. $6+1 \mathrm{w}$ and $6+5 \mathrm{w}$ are defined in Fig. 1. Determination of GSH was performed by a modified fluorometric method using OPT. Values are expressed as $\mu$ mole GSH per gram wet heart weight. Data represent the mean \pm SE of measurements from five separate rats. *, significant difference compared to saline control at $P<0.05$. 


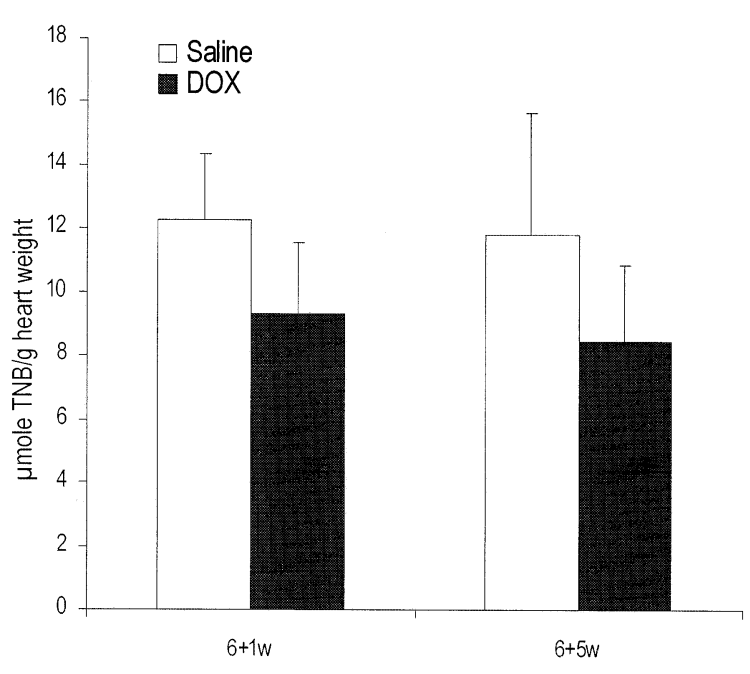

Fig. 3. Protein-SH content in heart tissue harvested from DOX/saline treated rats. Rats received 6 weekly injections of DOX $(2 \mathrm{mg} / \mathrm{kg})$ or saline. $6+1 \mathrm{w}$ and $6+5 \mathrm{w}$ are defined in Fig. 1. The protein-SH was determined spectrophotometrically at $412 \mathrm{~nm}$ by using 5,5'-dithiobis(2-nitrobenzoic acid). Values are expressed as $\mu$ mole TNB per gram wet heart tissue. Data represent the mean $\pm \mathrm{SE}$ of measurements from five separate rats.

chondria (Salazar and Van Houten, 1997; Yakes and Van Houten, 1997). Moreover, the pathways that are suggested to operate in the nucleus and mitochondria to repair oxidative DNA damage appear to be similar (Driggers et al., 1996). Accordingly, the accumulation of mtDNA adducts cannot be fully explained by the lack of repair of oxidized mtDNA. Indeed, in a previous study we found that $8 \mathrm{OHdG}$ adducts to cardiac mitochondria decrease to control values 2 weeks following a single injection of DOX (Palmeira et al., 1997), which suggests either the repair or replacement of oxidized mtDNA. The persistence of mtDNA adducts induced by DOX may, therefore, more likely represent a situation where the formation of $8 \mathrm{OHdG}$ is continuously stimulated and exceeds the limited capacity to repair these adducts.

Indeed, it is well established that DOX induces ROS generation due to the univalent reduction to its semiquinone free radical intermediate. However, it isn't likely that the persistent ROS generation 5 weeks after cessation of DOX treatment reflects redox cycling of the drug, because the residual doxorubicin is undetectable 7 days following the last injection (Solem et al., 1994, 1996). Therefore, we propose the alternate hypothesis that this persistent ROS generation occurs as a consequence of damage to the mitochondria caused by DOX. Indeed, it is well established that inhibition of the mitochondrial electron transport chain in vitro by antimycin A, results in a significant increase in the mitochondrial free radical production (Boveris and Chance, 1973; Turrens and Boveris, 1980; Turrens et al., 1982, 1985). In a recent study, Esposito et al. (1999) demonstrated that inhibition of oxidative phosphorylation by knocking out the adenine nucleotide translocator gene also results in increased ROS production and consequent damage to the mtDNA. Accordingly, it is feasible that repetitive DOX dosing causes some type of permanent impairment damage that leads to the persistent stimulation of ROS generation. For example, this could be a manifestation of the biogenesis of poorly coupled mitochondria (Esposito et al., 1999; Jeyaseelan et al., 1997; Papadopoulou et al., 1999 ) or the conversion of xanthine dehydrogenase to xanthine oxidase (Gustafson et al., 1991).

Because of its proximity to oxidative phosphorylation in the mitochondrial inner membrane, mtDNA is highly susceptible to oxidation by ROS and the oxidized DNA may directly contribute to mitochondrial genomic mutations. Unlike nuclear DNA, however, mtDNA encodes rRNAs, tRNAs and mitochondrial proteins with no nonsense sequences, except for a small region involved in the regulation of replication and translation of mtDNA. Thus, it is highly probable that any mutation in the mtDNA will result in a phenotypic response. This hypothesis is supported by a number of studies which show that damage to mtDNA causes a decrease in the levels of mitochondrial transcripts (Andreu et al., 1998; Calleja et al., 1993). Indeed, it has been demonstrated that DOX suppresses the expression of several mitochondrial genes, including genes encoding cytochrome c oxidase (Papadopoulou and Tsiftsoglou, 1996; Papadopoulou et al., 1999) and the adenine nucleotide translocator (Jeyaseelan et al., 1997). All these effects may lead to substantial alterations in the synthesis of mitochondrial 
proteins. Thus, following the last DOX dosing, it is possible that the newly synthesized dysfunctional mitochondria may continue to generate high levels of ROS, which in turn provide a continuous source of oxidation of mtDNA.

The overall antioxidant defense system is also an important factor determining the fate of DOXinduced ROS. It has been suggested that the occurrence of oxidative injury reflects stimulated generation of ROS that exceeds the capacity of antioxidant defense systems. GSH is the most important antioxidant against ROS. In agreement with previous reports that DOX treatment decreases GSH (Gustafson et al., 1993; Olson et al., 1980; Yin et al., 1998), we found that GSH is diminished in heart tissue from 6-week DOXtreated rats (c.a., 20\% less than control) and remained suppressed 5 weeks after discontinuation of DOX treatment (Fig. 2). This persistently lower GSH is consistent with the persistent ROS formation. However, there was no difference in protein-thiol content of heart tissue between DOX and saline-treated rats. This might be due to the effect of GSH, which though depleted, may still provide sufficient reduction of heart tissue protein-thiols against ROS-induced oxidation. In addition, there might be compensatory actions of other antioxidant systems in response to this increased oxidative stress in the cells. For example, it has been reported that DOX treatment increases metallothionein content and catalase activity in heart tissues (Yin et al., 1998).

In summary, we demonstrate a higher rate of ROS formation in cardiac myocytes isolated from rats following 6 weekly injections of DOX, and that this enhanced ROS generation persists for up to 5 weeks after the last injection. This correlates well with our previous finding of the cumulative and persistent oxidation of cardiac mtDNA. Associated with the sustained high rate of ROS formation is a persistent depression of GSH in heart tissue. The enhanced generation of ROS may directly damage mitochondria or alter mitochondrial genomic expression, thus altering the synthesis of proteins associated with the mitochondrial electron transport chain. All of these events might lead to a catastrophic bioenergetic dysfunction of mitochondria in a feed-forward cascade, eventually accounting for the cumulative and progressive pathogenesis of DOX-induced cardiomyopathy.

\section{Acknowledgements}

This work was supported by NIH grant HL58016. The authors thank Juline A. Smith for her help in isolating cardiac myocytes.

\section{References}

Andreu, A.L., Arbos, M.A., Perez-Martos, A., Lopez-Perez, M.J., Asin, J., Lopez, N., et al., 1998. Reduced mitochondrial DNA transcription in senescent rat heart. Biochem. Biophys. Res. Commun. 252, 577-581.

Boveris, A., Chance, B., 1973. The mitochondrial generation of hydrogen peroxide. General properties and effect of hyperbaric oxygen. Biochem. J. 134, 707-716.

Calleja, M., Pena, P., Ugalde, C., Ferreiro, C., Marco, R., 1993. Mitochondrial DNA remains intact during Drosophila aging, but the levels of mitochondrial transcripts are significantly reduced. J. Biol. Chem. 268, 18891-18897.

Driggers, W.J., Grishko, V.I., LeDoux, S.P., Wilson, G.L., 1996. Defective repair of oxidative damage in the mitochondrial DNA of a xeroderma pigmentosum group A cell line. Cancer Res. 56, 1262-1266.

Esposito, L.A, Melov, S., Panov, A., Cottrell, B.A., Wallce, D.C., 1999. Mitochondria disease in mouse results in increased oxidative stress. Proc. Natl. Acad. Sci. USA 96, 4820-4825.

Giulivi, C., Boveris, A., Cadenas, E., 1995. Hydroxyl radical generation during mitochondrial electron transfer and the formation of 8-hydroxydesoxyguanosine in mitochondrial DNA. Arch. Biochem. Biophys. 316, 909-916.

Gustafson, D.L., Swanson, J.D., Pritsos, C.A., 1991. Role of xanthine oxidase in the potentiation of doxorubicin-induced cardiotoxicity by mitomycin C. Canc. Commun. 3, 299-304.

Gustafson, D.L., Swanson, J.D., Pritsos, C.A., 1993. Modulation of glutathione and glutathione dependent antioxidant enzymes in mouse heart following doxorubicin therapy. Free Radic. Res. Commun. 19, 111-120.

Hissin, P.J., Hilf, R., 1976. A fluorometric method for determination of oxidized and reduced glutathione in tissues. Anal. Biochem. 74, 214-226.

Jeyaseelan, R., Poizat, C., Wu, H-Y., Kedes, L., 1997. Molecular mechanisms of doxorubicin-induced cardiomyopathy. J. Biol. Chem. 272, 5828-5832.

Kimura, T., Fujita, I., Itoh, N., Muto, N., Nakanishi, T., Takahashi, K., et al., 2000. Metallothionein acts as a 
cytoprotectant against doxorubicin toxicity. J. Pharmacol. Exp. Ther. 292, 299-302.

LeDoux, S.P., Wilson, G.L., Beecham, E.J., Stevnsner, T., Wasserman, K., Borhr, V.A., 1992. Repair of mitochondrial DNA after various types of DNA damage in Chinese hamster ovary cells. Carcinogenesis 13, 1967-1973.

Miyaki, M., Yatagai, K., Ono, T., 1977. Strand breaks of mammalian mitochondrial DNA induced by carcinogens. Chem. Biol. Interact. 17, 321-329.

Nicolay, K., De Kruijff, B., 1987. Effects of adriamycin on respiratory chain activities in mitochondria from rat liver, rat heart and bovine heart. Evidence for a preferential inhibition of complex III and IV. Biochim. Biophys. Acta 892, 320-330.

Niranjan, B.G., Bhat, N.K., Avadhani, N.G., 1981. Preferential attack of mitochondrial DNA by aflatoxin B1 during hepatocarcinogenesis. Science 215, 73-75.

Olson, R.D., MacDonald, J.S., Van Boxtel, C.J., Boerth, R.C., Harbison, R.D., Slonim, A.E., Freeman, R.W., Oates, J.A., 1980. Regulatory role of glutathione and soluble sulfhydryl groups in the toxicity of Adriamycin. J. Pharmacol. Exp. Ther. 215, 450-454.

Palmeira, C.M., Serrano, J., Kuehl, D.W., Wallace, K.B., 1997. Preferential oxidation of cardiac mitochondrial DNA following acute intoxication with doxorubicin. Biochim. Biophys. Acta 1321, 101-106.

Papadopoulou, L.C., Tsiftsoglou, A.S., 1996. Effects of hemin on apoptosis, suppression of cytochrome c oxidase gene expression and bone marrow toxicity induced by adriamycin. Biochem. Pharmacol. 52, 713-722.

Papadopoulou, L.C., Theophilidis, G., Thomopoulos, G.N., Tsiftsoglou, A.S., 1999. Structural and functional impairment of mitochondrial in adriamycin-induced cardiomyopathy in mice: suppression of cytochrome c oxidase II gene expression. Biochem. Pharmacol. 57, 481-489.

Praet, M., Ruysschaert, J.M., 1993. In-vivo and in-vitro mitochondrial membrane damages induced in mice by adriamycin and derivatives. Biochim. Biophys. Acta 1149, $79-85$.

Riddles, P.W., Blakeley, R.L., Zerner, B., 1979. Ellman's reagent:5,5'-dithiobis(2-nitrobenzoic acid) - a reexamination. Anal. Biochem. 94, 75-81.

Salazar, J.J., Van Houten, B., 1997. Preferential mitochondrial DNA injury caused by glucose oxidase as a steady genera- tor of hydrogen peroxide in human fibroblasts. Mutat. Res. 385, 139-149.

Serrano, J., Palmeira, C.M., Kuehl, D.W., Wallace, K.B., 1999. Cardioselective and cumulative oxidation of mitochondrial DNA following subchronic doxorubicin administration. Biochim. Biophys. Acta 1411, 201-205.

Solem, L.E., Henry, T.R., Wallace, K.B., 1994. Disruption of mitochondrial calcium homeostasis following chronic doxorubicin administration. Toxicol. Appl. Pharmacol. 129, 214-222.

Solem, L.E., Heller, L.J., Wallace, K.B., 1996. Dose-dependent increase in sensitivity to calcium-induced mitochondrial dysfunction and cardiomyocyte cell injury by doxorubicin. J. Mol. Cell Cardiol. 28, 1023-1032.

Stairs, P.W., Guzelian, P.S., Van Tuyle, G.C., 1983. Benzo[a]pyrene differentially alters mitochondrial and nuclear DNA synthesis in primary hepatocyte cultures. Res. Commun. Chem. Pathol. Pharmacol. 42, 95-106.

Turrens, J.F., Boveris, A., 1980. Generation of superoxide anion by the NADH dehydrogenase of bovine heart mitochondria. Biochem. J. 191, 421-427.

Turrens, J.F., Freeman, B.A., Levitt, J.G., Crapo, J.D., 1982. The effect of hyperoxia on superoxide production by lung submitochondrial particles. Arch. Biochem. Biophys. 217, $401-410$

Turrens, J.F., Alexandre, A., Lehninger, A.L., 1985. Ubisemiquinone is the electron donor for superoxide formation by complex III of heart mitochondria. Arch. Biochem. Biophys. 237, 408-414.

Wang, G.-W., Kang, Y.J., 1999. Inhibition of doxorubicin toxicity in cultured neonatal mouse cardiomyocytes with elevated metallothionein levels. J. Pharmacol. Exp. Ther. 288, 938-944.

Wu, H-Y., Kang, Y.J., 1998. Inhibition of buthionine sulfoximine-enhanced doxorubicin toxicity in metallothionein overexpressing transgenic mouse heart. J. Pharmacol. Exp. Ther. 287, 515-520.

Yakes, F.M., Van Houten, B., 1997. Mitochondrial DNA damage is more extensive and persists longer than nuclear DNA damage in human cells following oxidative stress. Proc. Natl. Acad. Sci. USA 94, 514-519.

Yin, X., Wu, H., Chen, Y., Kang, Y.J., 1998. Induction of antioxidants by adriamycin in mouse heart. Biochem. Pharmacol. 56, 87-93. 